\title{
Construyendo comunes para el derecho a la ciudad a través de la innovación social colectiva en la distribución y consumo: explorando un marco conceptual y el caso de Valencia
}

\begin{abstract}
Sergio Belda-Miquel. Universitat de València, Valencia, España.
Victoria Pellicer-Sifres. Universitat Politècnica de València, Valencia, España.

Alejandra Boni. Ingenio (csic-Universitat Politècnica de València), Valencia, España.

RESUMEN | En la actual crisis urbana, el nuevo municipalismo reclama la reconstrucción de comunes urbanos para avanzar en el derecho a la ciudad. Seńala al respecto la importancia de los movimientos sociales y de la acción pública municipal para generar procesos de innovación social "desde abajo". Sin embargo, no se ha explorado suficientemente la literatura sobre innovación social con miras a comprender la construcción de comunes urbanos. Por ello, este trabajo propone, a partir de la literatura sobre innovación social de base, un marco para explorar prácticas de generación de comunes desde la ciudadanía, así como políticas municipales que pueden articularse para apoyarlas. El marco se emplea para explorar, con métodos cualitativos, iniciativas en Valencia (Estado espańol), en los sectores agroalimentario y energético. Los resultados revelan el potencial y la diversidad de las iniciativas de generación de comunes urbanos para el derecho a la ciudad, así como tensiones y contradicciones que pueden emerger.
\end{abstract}

PALABRAS CLAVE $\mid$ movimientos sociales, transformaciones socioterritoriales, consumo.

ABSTRACT | In the present urban crisis, the new municipalism claims for the rebuilding of the urban commons for advancing the right to the city. In this sense, some voices point to the essential role of social movements and local policy action to promote bottom-up social innovation processes. Nevertheless, the use of the literature on social innovation to understand the construction of urban commons remains underexplored. For this reason, this work proposes, drawing on the literature of grassroots innovation, a framework to explore practices of the generation of commons from the citizenship, as well as local policies to support them. This frame is applied in the exploration, using qualitative methods, of initiatives in Valencia (Spain) in the agro-food and energy sectors. Results reveal the potential and diversity of the initiatives building urban commons for the right to the city and emerging tensions and contradictions.

KEYWORDS | social movements, socio-territorial transformations, consumption. 


\section{Crisis urbana, construcción del común, derecho a la ciudad e innovación social desde las comunidades}

Las ciudades son el escenario central de los nuevos procesos de desposesión, precarización y desigualdad (Harvey, 2004). En este contexto, se ha recuperado la idea del derecho a la ciudad, cada vez más presente en los debates políticos y académicos a nivel global y local (Belda-Miquel et al., 2016; Mayer, 2009; Purcell, 2014). Estos debates abordan cuestiones acerca de qué estrategias son necesarias, qué actores han de estar en el centro o qué alianzas han de establecerse para construir este derecho (Belda-Miquel et al., 2016; Purcell, 2013).

La corriente más radical en este debate recupera las ideas originales de Lefebvre (Belda-Miquel et al., 2016; Mayer, 2009), que ponen en el centro la necesidad de generar prácticas de autogestión para transformar las relaciones de poder que producen la ciudad y superar tanto la lógica del Estado como la del mercado (Lefebvre, 2003[1970]). En esta corriente se sitúan las propuestas del nuevo municipalismo, que se sostendrían en dos ejes fundamentales (Blanco et al., 2018; Subirats \& García, 2015). Por un lado, ponen en el centro la acción de los movimientos sociales y comunitarios para crear prácticas, lógicas y universos alternativos que puedan hacer efectivo el derecho a la ciudad "desde abajo". Diversos estudios en los últimos años han abordado la capacidad colectiva de transformación en la ciudad a través de prácticas de autogestión basadas en la cooperación y la ayuda mutua, particularmente en el contexto espańol (Castells \& Hlebik, 2016). Por otro lado, el nuevo municipalismo otorga una importancia fundamental al nivel local y a las políticas públicas municipales para la construcción del derecho a la ciudad. Se reclama para ello una acción pública renovada, multidimensional, integrada, abierta y participativa (Blanco \& Gomà, 2016; Brown, 2013; Fernández, 2018). Lo local se considera el lugar privilegiado desde el cual plantear nuevas lógicas de relación y nuevas formas de reconocimiento entre la administración y los movimientos de base. Así se superan planteamientos dicotómicos para abrir vías de apoyo institucional a procesos "desde abajo" (Blanco \& Gomà, 2016).

Estos debates sobre las relaciones entre prácticas de autogestión y acción pública municipal para el derecho a la ciudad se han concretado en el debate sobre la recuperación de lo común en la ciudad (Castro-Coma \& Martí-Costa, 2016). Según Harvey (2004), los problemas urbanos contemporáneos se deben a una nueva estrategia de acumulación del capitalismo, la "acumulación por desposesión" (Harvey, 2004). Sería un nuevo proceso de apropiación de los recursos de la ciudad por parte del capital, que se manifiesta en cuestiones como la privatización del espacio público, el deterioro de los servicios, la segregación o la desigualdad. En este sentido, las ciudades serían objeto de operaciones extractivas de los comunes urbanos (Castro-Coma \& Martí-Costa, 2016; Gago \& Pérez, 2014). Por ello, para estos autores los comunes urbanos tienen un lugar fundamental como lugares de resistencia y de transformación para reivindicar y avanzar hacia el derecho a la ciudad (Harvey, 2004). Se trata, así, de reclamar y recuperar los bienes comunes; de generar prácticas de defensa, generación y gestión de recursos, bienes y servicios de 
manera colectiva y democrática, fuera de la lógica del capital y del mercado y de la lógica burocrática del Estado.

Por comunes urbanos nos referimos, siguiendo a Bollier (2014), a "sistemas sociales que administran de forma comunitaria bienes y recursos, de modo que se preservan valores y una identidad comunes (...), sistemas autoorganizados y gestionados por la propia comunidad, con ninguna o muy poca intervención del estado o del mercado (...) y que crean una riqueza que pasa, sin reducirse, a nuestros hijos" (p. 175). Esto puede incluir bienes tanto materiales (por ejemplo, infraestructuras, ecosistemas urbanos, suelo, agua, vivienda o producción de alimentos) como inmateriales (seguridad, cuidados, cultura...). Pueden ser también bienes producidos (como la vivienda o nuevos bienes culturales) o heredados de tiempo atrás (como los bienes naturales o la propia lengua y el patrimonio cultural) (Agyeman et al., 2016).

La literatura sobre comunes urbanos ha abordado numerosos casos: de la gestión de la seguridad o el suelo a los sistemas de distribución de agua y electricidad; de la gobernanza de ecosistemas naturales urbanos y espacios públicos a prácticas de autogestión en múltiples sectores (Blanco et al., 2018; Castells \& Hlebik, 2016, Castro-Coma \& Martí-Costa, 2016). Para el caso español, Castells y Hlebik (2016) muestran prácticas en muy distintos campos: mercados campesinos, cooperativas de producción o consumo, bancos de semillas o comedores cooperativos en el ámbito agroalimentario; en el ámbito de la energía, cooperativas y grupos de autoproducción; monedas sociales, banca ética y sistemas de intercambio comunitario; cooperativas de viviendas y centros sociales; grupos de crianza compartida en el ámbito de los cuidados; redes de librerías, grupos de autoaprendizaje, universidades libres y museos abiertos en el ámbito de la cultura, etcétera.

En esta literatura aparece un concepto fundamental para abordar la emergencia y desarrollo de estas prácticas: la innovación social. Las prácticas de generación de comunes pueden verse, para diversos autores, como prácticas de innovación social. Además, la literatura habla de la importancia de apoyar la innovación social desde las instituciones municipales (Subirats \& García, 2015), en tanto generan comunes urbanos.

La aplicación del concepto de innovación social para abordar la producción de comunes urbanos y el derecho a la ciudad ha sido también criticada. Se ha apuntado que puede despolitizar los análisis y generar la captura de estos procesos por la agenda neoliberal (Miró, 2015). Sin embargo, el concepto resulta atractivo para diversos autores: las prácticas de creación de comunes serían innovadoras en tanto generan espacios al margen de las lógicas dominantes, lo cual conduce a la reflexión sobre la forma en que surgen y se desarrollan como nichos alternativos (Marques et al., 2018). Además, hablar de innovación social permite apelar a la dimensión creadora, desde la disidencia, de los movimientos sociales (Blanco et al., 2018).

Varios trabajos han abordado las conexiones entre construcción de comunes para el derecho a la ciudad e innovación social, para distintos ámbitos y sectores (Andretta et al., 2015; Blanco \& Gomà, 2016; Hernández et al., 2017; Moulaert, 2010; Subirats \& García, 2015). Sin embargo, estos trabajos tienen un enfoque principalmente descriptivo. Aunque esto no los priva en absoluto de interés, no parece existir una propuesta teórica suficientemente desarrollada que permita 
abordar de forma más comprensiva los procesos, estrategias y dinámicas de innovación social para la construcción de comunes. Tampoco los tipos de políticas que se pueden articular, especialmente a nivel local, para visibilizar, apoyar o promover estos procesos. Estas limitaciones pueden deberse a que no se han explorado de manera consistente las conexiones entre debates y conceptos de las literaturas sobre comunes urbanos y sobre innovación. Como señalan Castro-Coma y Martí-Costa (2016), "el estudio de los comunes urbanos desde una perspectiva compleja requiere de una nueva agenda de investigación para el estudio de aquellas prácticas sociales y comunitarias que desbordan lo estatal (...) para hacer efectivo el derecho colectivo a la ciudad" (p. 148).

Los estudios sobre innovación han puesto el foco esencialmente en los procesos de cambio en el marco del mercado o de la acción pública. Sin embargo, existe una corriente que, en torno a la idea de grassroots innovation -innovación social desde la base, "desde abajo" o colectiva- ha tratado de entender los procesos de innovación generados autónomamente por la propia ciudadanía. Se definen como procesos promovidos por "redes de activistas y organizaciones que generan nuevas soluciones frente a los retos sociales y de sostenibilidad, sobre la base de los valores y visiones locales" (Seyfang \& Smith, 2007, p. 585). Esta literatura ha estudiado cómo estas iniciativas nacen y se desarrollan como nichos de innovación en los márgenes de los sistemas existentes (Ornetzeder \& Rohracher, 2013); sus dinámicas de éxito o fracaso (Feola \& Nunes, 2014); o cómo difunden sus prácticas y pueden influenciar o sustituir sistemas (Hoppe et al., 2015). Tales cuestiones se han explorado en casos en distintas áreas y sistemas: energía (Hargreaves et al., 2013), alimentación (Pellicer-Sifres et al., 2017), vivienda (Seyfang \& Smith, 2007), moneda social (Seyfang \& Longhurst, 2013), etcétera. Se trata de materias y sectores muy relevantes para comprender procesos comunitarios de producción de comunes.

Desde estas premisas, el trabajo tiene el objetivo, en primer lugar, de proponer un marco de análisis que permita comprender el desarrollo de prácticas y estrategias de innovación social que estarían generando comunes urbanos para el derecho a la ciudad, así como las políticas que pueden articularse para apoyarlos. En segundo lugar, se aplica el marco para explorar procesos concretos de innovación social en dos sectores clave en la ciudad de Valencia. El enfoque del trabajo es puramente exploratorio: no se trata de comprender en profundidad los casos, sino de obtener, del análisis empírico, elementos que permitan ilustrar el alcance y límites del marco propuesto, así como continuar identificando cuestiones relevantes para nuestro objeto de estudio.

En la siguiente sección proponemos el marco teórico del trabajo. A continuación, explicamos la metodología para el análisis de los casos, que son contextualizados y analizados, antes de cerrar con algunas ideas finales sobre los objetivos del trabajo.

\section{Marco de análisis}

\section{Articular el derecho a la ciudad a través de la construcción de los comunes}

Desde el nuevo municipalismo, autores como Blanco et al. (2018) y Blanco y Gomà (2016) consideran que el derecho a la ciudad puede ser alcanzado a través de la construcción de comunes urbanos. El horizonte de ciudad radicalmente democrático 
al que se refiere tal noción conecta con las ideas centrales de Lefebvre (2003 [1970]) sobre el derecho a la ciudad. En primer lugar, construir este derecho implicaría reorientar la producción de la ciudad a las necesidades de las personas, situar el valor de uso por encima del valor de cambio (Purcell, 2014). En segundo lugar, sería un proceso de generación de autogobierno del espacio urbano, de toma de las decisiones que afectan a la comunidad por parte de las personas que habitan la ciudad, independientemente de su estatus legal (Purcell, 2013). En tercer lugar, es un proyecto de nuevo modelo de sociedad, más allá de la lógica del mercado y del Estado (Lefebvre, 2003 [1970]). Finalmente, se puede mencionar la dimensión pedagógica emancipadora, ya que los habitantes se transforman mientras producen y transforman la ciudad, en procesos permanentes de aprendizaje (Belda-Miquel et al., 2016; Purcell, 2013). Todas estas dimensiones - la desmercantilización, la autoorganización, la superación de la lógica mercantil y burocrática, la conexión entre transformación individual y colectiva- son consustanciales a los comunes urbanos.

Si los mencionados son los elementos fundamentales del horizonte de derecho a la ciudad buscado, el nuevo municipalismo sitúa la esfera local como espacio instituyente de esos cambios. En este sentido se identifican dos aspectos clave. Por un lado, estaría la centralidad de las prácticas ciudadanas, esto es, prácticas de innovación social orientadas hacia una agenda urbana creadora del común, en las que los movimientos sociales no renuncian a la disidencia sino que, a partir de la misma, ejercen la creación (Blanco et al., 2018). Estas iniciativas se movilizan para defender y hacer efectivos derechos concretos y generan espacios de autogestión en distintos sectores y ámbitos.

Por otro lado, estaría el rol de la acción pública en el ámbito local y la importancia de generar una esfera pública compartida (Blanco et al., 2018). Castro-Coma y Martí-Costa (2016) explican que

aunque las prácticas de autogestión pueden favorecer modos más democráticos de participación a nivel local, el papel del Estado no puede ser ignorado. En esta línea se hace necesario explorar las alianzas comunales-estatales que se pueden desarrollar en cada contexto y ámbito particular, ampliando lo que se considera como público más allá de lo estrictamente estatal. (p. 147)

Pueden existir múltiples formas para la generación de esta esfera pública, desde la presencia institucional de actores sociales a alianzas puntuales o sostenidas y a procesos de coproducción de acciones y políticas (Blanco et al., 2018). En cualquier caso, las formas de interacción entre lo público y lo comunitario van a ser siempre tensas, complejas y cambiantes, sometidas a la generación permanente de nuevas estrategias y equilibrios.

Para comprender mejor tanto las dinámicas de innovación social como las formas de respuesta y de interacción desde la esfera de lo público, exploramos elementos de la literatura sobre innovación en las siguientes dos secciones. 


\section{La innovación social desde la base: procesos para desarrollar y transformar los sistemas existentes}

La literatura sobre innovación social desde la base ha abordado los procesos de innovación impulsados por la ciudadanía autoorganizada que generan sistemas alternativos de producción y consumo. Esta literatura señala algunas características clave de estos procesos (Seyfang \& Smith, 2007): se basan en la economía social; están orientados por necesidades sociales y por ideología (no por beneficio económico); obtienen sus recursos esencialmente del trabajo y aportaciones voluntarias y del intercambio, aunque pueden obtenerse de subvenciones o de actividad comercial limitada y no lucrativa; pueden ser promovidos por colectivos muy variados, tales como organizaciones vecinales, de voluntarios, cooperativas, empresas sociales, grupos informales, etcétera; y operan en muy distintos sectores (Hargreaves et al., 2013; Hossain, 2018; Seyfang \& Longhurst, 2013).

Desde los enfoques de transiciones sociotécnicas y de gestión estratégica, estas prácticas constituyen nichos, esto es, espacios alternativos donde se dan iniciativas que generan prácticas distintas a las dominantes, en los que se ensayan y prefiguran lógicas y modelos diferentes a los hegemónicos (Geels, 2002). Se dan prácticas alternativas al llamado régimen de un sistema (por ejemplo, el alimentario o el energético), esto es, distintas al conjunto de normas, rutinas cognitivas, comportamientos compartidos, capacidades y competencias, estilos de vida, prácticas diarias, acuerdos institucionales, y en general las reglas extendidas y comúnmente aceptadas por las que se rigen los sistemas y por las que permanecen estables (Geels, 2010).

Las iniciativas de producción de comunes urbanos presentarían así las características de procesos de innovación social desde la base: prefiguran y construyen alternativas; operan en un primer momento de forma paralela y alternativa a las formas hegemónicas de gestión y acceso a bienes y servicios; y aspiran a transformar los sistemas. Esta literatura ofrece elementos para aproximarnos a aquellos caminos por los cuales iniciativas de innovación de base encuentran oportunidades para cambiar los sistemas dominantes; a las estrategias que desarrollan; y a la forma en que buscan crecer y consolidarse (Seyfang \& Haxeltine, 2012).

En primer lugar, nos referimos al contexto y oportunidades para el desarrollo $y$ crecimiento de iniciativas de innovación social desde la base. En la literatura, distintos autores señalan que los nichos de innovación social pueden influenciar o incluso sustituir al régimen en momentos de crisis y fuertes presiones estructurales (Geels, 2002). Los regímenes pueden verse tensionados y sacudidos por el llamado panorama más amplio: procesos sociales (por ejemplo, intensas movilizaciones), económicos (crisis económicas), políticos (momentos de apertura y cambio político e institucional, o momentos de descrédito de sistemas existentes) o ambientales (las presiones que introduce el cambio climático). Cuando ocurre, pueden abrirse ventanas de oportunidad para que los nichos puedan desarrollarse, influir y eventualmente sustituir al régimen si están suficientemente maduros (Geels, 2002, 2010).

En segundo lugar, podemos hablar de las estrategias que desarrollan las iniciativas. Sobre la base de las teorías sobre gestión estratégica de nichos, varios autores apuntan que existen tres procesos básicos en el desarrollo de nichos (Schot \& Geels, 2008): 
- La articulación de expectativas y visiones. Serían procesos permanentes de generación de visiones alternativas a las dominantes, así como de conexión con las expectativas de las personas para comprometerlas. Las iniciativas innovadoras (re)articulan permanentemente miradas, discursos, objetivos y visiones, lo cual modela la capacidad de movilización.

- La construcción de redes. Las iniciativas buscan construir redes sociales, interactuar y relacionarse con distintos actores para conocer con más profundidad el propio entorno; seguir adaptándose y transformándose; y para inspirarse, ofrecerse apoyo mutuo o formar nuevas iniciativas, entre otras posibilidades.

- La profundización y el aprendizaje. En las iniciativas de innovación de base se dan permanentemente procesos de aprendizaje, profundización y articulación internas. Por ejemplo, acciones para mantener viva la experimentación y la reflexión; para profundizar en la movilización y la participación; o para manejar las limitaciones y oportunidades del contexto.

En tercer lugar, la teoría sobre gestión de nichos habla de los patrones de difusión de ideas y prácticas alternativas. Siguiendo a Seyfang y Haxeltine (2012), y a otros autores, las iniciativas pueden crecer mediante tres procesos diferentes:

- A través de la replicación. Esto es, con la multiplicación de distintas iniciativas dentro del nicho, lo cual produce cambios agregativos en el sistema a través de la suma de una multiplicidad de iniciativas más pequeńas.

- A través del crecimiento de escala. Esto es, atrayendo más participantes y volumen de actividad a las iniciativas existentes, que incrementan así su tamaño e impacto.

- A través de la traslación, esto es, mediante el flujo de las ideas, que pueden pasar de unas iniciativas a otras dentro del nicho, o que pueden pasar al régimen e incorporarse a las prácticas dominantes del sistema.

En su diversidad de estrategias y prácticas, las iniciativas tienen también distintas visiones sobre el rol de la acción pública: pueden ser recelosas y centradas en operar plenamente al margen del Estado; de apertura a recibir cierto respaldo puntual o más continuado (apoyo técnico o logístico o para ganar visibilidad o legitimidad, por ejemplo); o centradas en solicitar cambios normativos y de políticas; o una combinación de las anteriores (Suriñach, 2017).

\section{Políticas para la innovación social desde la base}

Dadas las distintas estrategias, visiones y demandas de las iniciativas de base, deben ser variadas las políticas que pueden responder a las mismas. La literatura sugiere que es precisamente la combinación de políticas lo que puede dar fuerza y sentido a una acción pública que tenga en su centro los nichos de iniciativas de innovación social desde la base. Apoyándonos en el trabajo de distintos autores (Blanco et al., 2018; Kivimaa \& Kern, 2016; Surińach, 2017), proponemos una clasificación en tres tipos de políticas.

En primer lugar, políticas de protección de nichos. Se trataría de acciones destinadas a generar espacios seguros para las iniciativas a fin de evitar o limitar la presión externa 
sobre ellas, o a dar acompańamiento en los momentos iniciales, cuando las iniciativas lo demandan. Por ejemplo: dar protección legal y generar espacios regulatorios específicos; generar excepciones fiscales; facilitar espacios, logística y acceso a conocimiento; evitar la presión y persecución en situaciones ambiguas normativamente.

En segundo lugar, políticas de apoyo o consolidación. Se trataría de acciones para acompañar en la maduración, desarrollo y refuerzo de las iniciativas cuando lo solicitan. Por ejemplo, apoyar la realización de encuentros, la creación de redes y el intercambio de conocimiento; dar legitimidad, apoyo institucional y visibilidad; facilitar el acceso a nuevos públicos; ofrecer recursos logísticos, técnicos u otros que se soliciten, etcétera.

En tercer lugar, políticas para el empoderamiento, escalado y expansión. Se trataría de acciones orientadas a facilitar el crecimiento de nichos e iniciativas, sin que esto suponga la renuncia a principios y valores. Por ejemplo, realizar cambios regulatorios; emplear la compra pública; facilitar la construcción y acceso de iniciativas locales a redes en escalas más amplias; cocrear las propias estrategias y políticas públicas, etcétera.

Todas estas acciones pueden ser muy diversas y darse en distintas dimensiones: en la creación y difusión de conocimiento; en el desarrollo de nuevas formas, canales, infraestructuras, modelos de gestión y esquemas de producción, intercambio o consumo; en el ámbito de las relaciones entre actores; en la generación y el uso de tecnologías, etcétera. En cualquier caso, articular políticas que pongan en el centro la promoción de iniciativas innovadoras que están generando comunes urbanos requieren de una acción compleja, combinada, contextual y conectada con las demandas reales y explícitas de las iniciativas de base.

\section{Innovación social desde la base para la construcción de los comunes}

Conectando los conceptos presentados, proponemos un marco propio para comprender el contexto, estrategias, desarrollo y políticas en relación con procesos de generación de comunes urbanos entendidos como procesos de innovación social desde la base (Figura 1).

FIGURA I | Innovación social desde la base y producción de comunes para el derecho a la ciudad

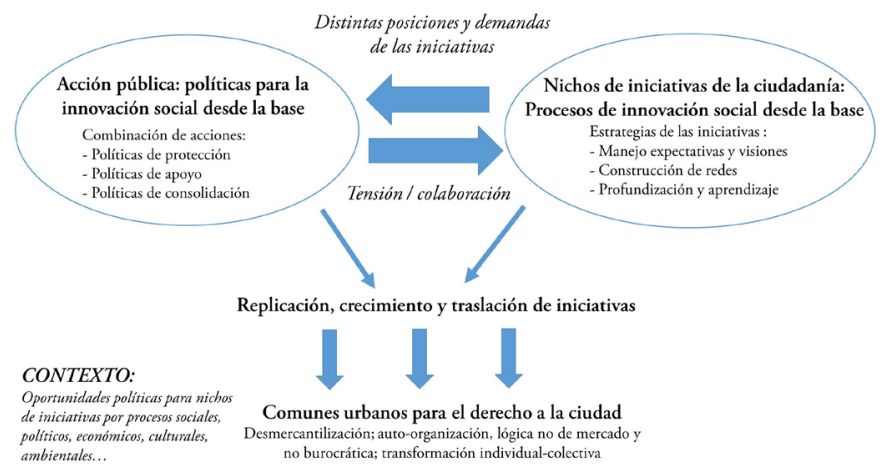

FUENTE: ELABORACIÓN PROPIA 


\section{Metodología}

Se han seleccionado como casos dos nichos de iniciativas en dos sectores en la ciudad de Valencia. En primer lugar, en el sector alimentario, el nicho de iniciativas de canales alimentarios alternativos. En segundo lugar, en el sector de la energía, el nicho de canales y formas de consumo alternativos de energía. La ciudad, los sectores y los nichos se han seleccionado por criterios de accesibilidad y de relevancia para la teoría propuesta y para obtener implicaciones prácticas (Miles \& Huberman, 1984). Por ello, los casos se caracterizan por lo siguiente: son sectores clave para la sostenibilidad y centrales en los debates internacionales, estatales y locales; existe un buen número de iniciativas en ambos nichos en la ciudad, accesibles a la investigación; los dos nichos presentan diferencias interesantes, tanto en relación con la propia naturaleza de los bienes, su regulación y su gobernanza, como respecto de la madurez y coordinación de iniciativas en el nicho.

Para la obtención de información se realizaron entrevistas semiestructuradas a distintos actores: personas expertas (una para el caso de energía y dos para el de alimentación); miembros de iniciativas del nicho (cuatro para energía y seis para alimentación, una por cada iniciativa); personas vinculadas a la política pública en el ámbito local (una a la política energética, dos a políticas de alimentación y una a políticas de innovación). Además, se realizó investigación participante mediante la asistencia a foros y espacios de encuentro de estos nichos, organizados tanto de forma autónoma como por el Ayuntamiento de Valencia.

Para el procesamiento de información, se ha empleado un enfoque deductivo a partir de las categorías del marco propuesto. Para cada una de esas categorías se buscaron patrones de similitud y de diferencia dentro de las iniciativas de cada nicho, y entre los dos nichos casos de estudio. Se partió de presunciones propias de los paradigmas interpretativista y crítico, siguiendo la clasificación de Lincoln et al. (2011). Se considera el conocimiento mediado por las perspectivas e interacciones, pero también por las posiciones y relaciones de poder. La estrategia de investigación se orienta a comprender los significados, marcos y acciones de distintos actores clave del fenómeno bajo estudio, en su propio contexto geográfico, social, económico y político (Corbetta, 2003). La orientación es esencialmente exploratoria, ya que se trata de indagar en el alcance y potencial del marco propuesto y de sugerir cuestiones clave en las que profundizar.

\section{Casos de estudio: descripción y contexto}

\section{Iniciativas en el ámbito agroalimentario}

Valencia ha visto, como otras ciudades, el surgimiento de múltiples experiencias alternativas al régimen agroalimentario industrial dominante. Como señalan Seyfang y Smith (2007), este régimen se basa en el uso intensivo de tecnología (agroquímicos, biotecnología, tratamientos hormonales, etc.); en la producción intensiva, especializada a nivel global y sostenida en grandes operaciones comerciales (con frecuencia puramente especulativas); en la dependencia respecto de insumos industriales controlados por unos pocos actores; en el control de los canales 
de comercialización por unas pocas grandes empresas; en la compra en grandes superficies, con fuerte presencia de productos envasados y procesados. El modelo se basa esencialmente en conocimiento científico estandarizado, monopolizado por la gran empresa y grandes centros de investigación.

El régimen descrito está fuertemente presionado por su impacto en el cambio climático, por los cambios en los patrones alimentarios, por escándalos relacionados con la seguridad alimentaria y por la persistencia de los problemas alimentarios globales y el avance de nuevos (como la obesidad). Para múltiples voces críticas, este sistema estaría también en la base de la desaparición de la agricultura familiar y de su valor productivo, histórico, cultural y ambiental. En este contexto, en ciudades y áreas periurbanas, múltiples iniciativas estarían buscando construir sistemas basados en la producción y comercialización sostenible, local, agroecológica, justa para todos los actores, basada en productos de temporada, en la minimización de intermediarios y en la confianza entre productor y consumidor (Goodman et al., 2012). Tal tendencia global se daría con fuerza en el contexto español (Díaz Escobar, 2014).

En el caso de Valencia, se ha incrementado recientemente el número de iniciativas de canales alternativos de producción, distribución y consumo alimentarios, que han ganado madurez y visibilidad (véase, por ejemplo, Moragues-Faus, 2017). Estas iniciativas plantean formas y estrategias distintas, pero se basarían en principios comunes, como los señalados: sostenibilidad, agroecología, relocalización de sistemas, apoyo a la agricultura familiar, revitalización de espacios urbanos o periurbanos (Pellicer-Sifres et al., 2017). Todas se abastecen principalmente de producciones de agricultores o cooperativas agroecológicos del área periurbana de Valencia. Se ha analizado una muestra de iniciativas que pretende capturar esta diversidad, cuyas principales características se exponen en la Tabla 1. 


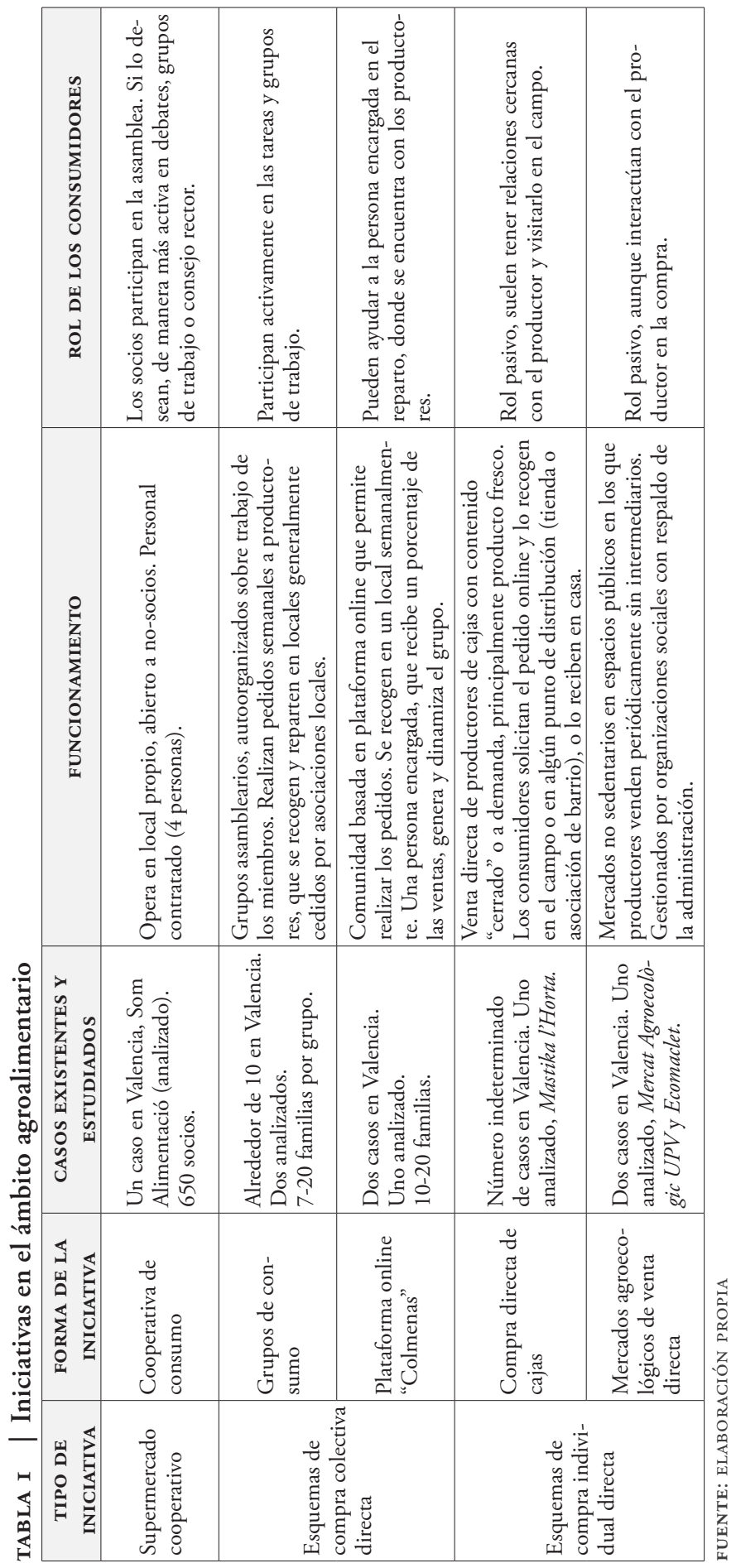




\section{Iniciativas en el ámbito energético}

En el ámbito de la energía, han emergido también numerosas iniciativas orientadas a un modelo energético más justo y sostenible (Riutort Isern, 2015; Xarxa per la Sobirania Energètica, 2018). El modelo dominante se caracteriza por estar fuertemente centralizado - cinco compañías controlan el 80\% de la producción y el $95 \%$ de la comercialización (APPA [Asociación de Empresas de Energías Renovables], 2013)-; por ser muy dependientes de fuentes no renovables (Sánchez-Herrero, 2014) y generar precios de consumo que están entre los más altos de Europa (Sánchez-Herrero, 2014). El precio se fija diariamente en subastas de energía en las que productores y comercializadores se encuentran (solo se puede comercializar energía comprándola en la subasta). Las críticas por las prácticas oligopólicas, por la opacidad, por el uso de "puertas giratorias", por las fuertes alzas de precios o por la propia insostenibilidad ambiental del modelo, junto con los compromisos adquiridos por las administraciones en la reducción de emisiones, han generado una importante ventana de oportunidad para las iniciativas por un modelo energético alternativo (Capellán-Pérez et al., 2018). Con todo, hasta octubre de 2018, las regulaciones gravaban y complicaban enormemente la autoproducción de energía y generaban una casi total dependencia de la red centralizada y sus actores dominantes.

En la investigación se ha abordado casos en Valencia que, moviéndose en los márgenes que permite la normativa, tratan de generar un modelo alternativo, más democrático y sostenible de producción, distribución y consumo de energía. En la Tabla 2 se describen estos casos, que presentan gran diversidad. 


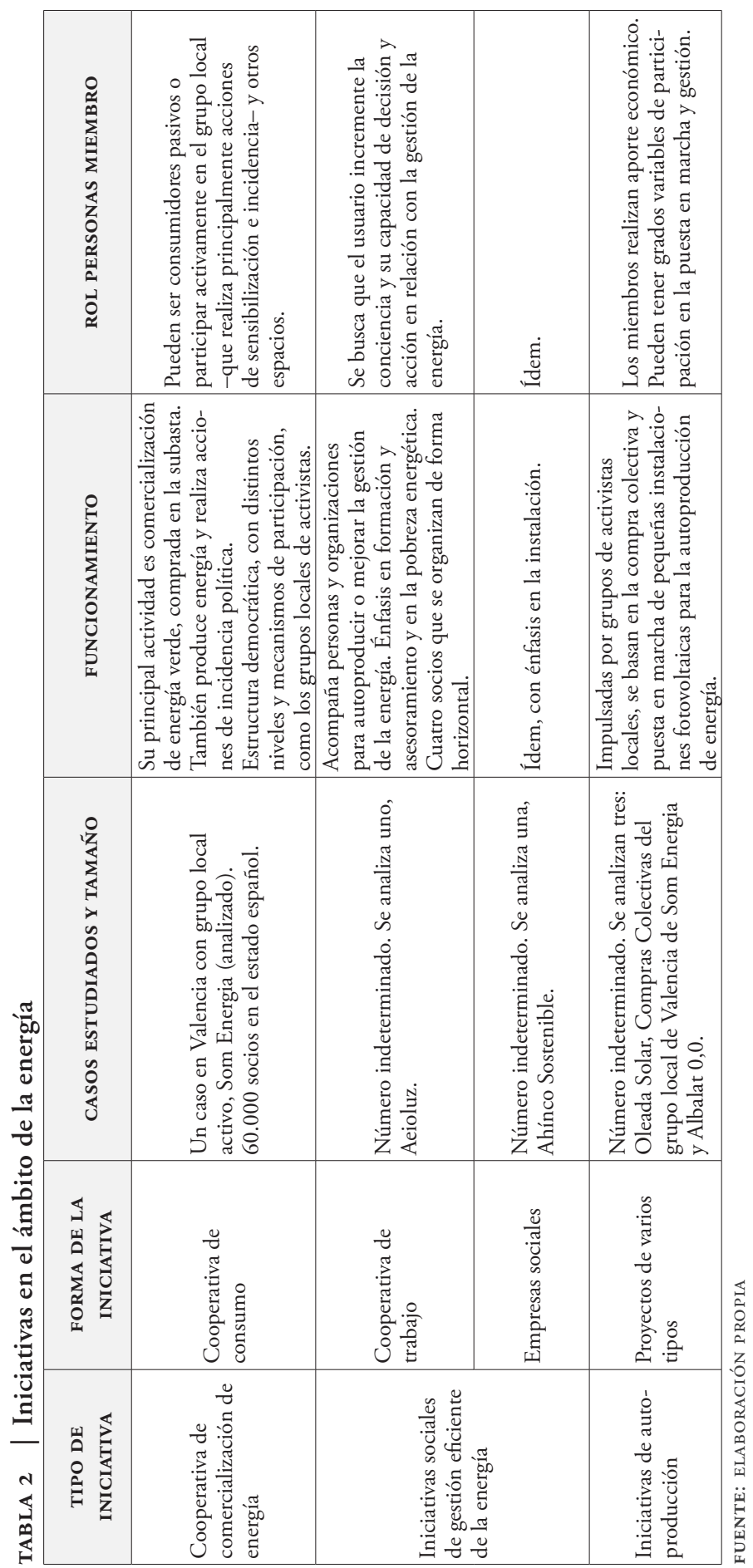




\section{Discusión y resultados}

\section{Las estrategias de las iniciativas}

Los resultados revelan la existencia de estrategias distintas en la acción de las iniciativas en los nichos. En primer lugar, sobre la articulación de expectativas y visiones, observamos que existen lenguajes, conceptos y visiones comunes en cada nicho y entre los nichos casos de estudio. Dentro de los nichos, algunas iniciativas tienen una estrategia más específica que busca movilizar a ciertos colectivos.

Las iniciativas en el nicho de la energía tienen como visión común la transformación del modelo energético hacia un modelo más sostenible y democrático, respetuoso con el medioambiente y gobernado por la propia ciudadanía. Los casos se refieren a cuestiones como "impulsar un modelo energético en manos de la ciudadanía", "romper el oligopolio" y "participar de un movimiento social transformador" (Som Energia, 2020). Esto conecta con elementos del derecho a la ciudad señalados: la energía como bien común que se requiere desmercantilizar, la autoorganización de la ciudadanía y la importancia de la participación para la transformación individual y colectiva. Aunque diversas personas y documentos consideran la creación de un modelo radicalmente descentralizado y ciudadano como el escenario más deseable, buena parte de las iniciativas asumen que la realidad regulatoria y la falta de recursos lo hacen imposible. Por ello, plantean intervenciones menos ambiciosas, orientadas a movilizar a distintos públicos: por ejemplo, Som Energia, que se centra en la comercialización de energía de fuentes renovables. Dadas las dificultades de autoproducción, otras iniciativas se centran en asesorar y formar para gestionar la energía de forma más consciente, como Aeioluz y Ahínco Sostenible. Otras intentan operar con las limitaciones existentes y se centran en la compra colectiva de instalaciones de renovables (con frecuencia para evitar que sean compradas por los actores dominantes), como los casos mencionados en la Tabla 2.

En paralelo, todas estas iniciativas participan de distintos espacios y plataformas, orientados a generar cambios en las políticas que permitan avanzar en la idea de energía como bien común, faciliten la autoproducción y rompan el monopolio energético, como es el caso de la Plataforma por un Nuevo Modelo Energético. Además, en todas las iniciativas se avanza en la desmercantilización, en tanto que, bajo distintas figuras jurídicas y esquemas organizativos, funcionan sin ánimo de lucro.

En el nicho de iniciativas de alimentación se dan cuestiones similares: su visión es la de un sistema agroalimentario controlado por productores y consumidores, también descentralizado, desmercantilizado y radicalmente democrático. Varias de las alternativas se refieren a cuestiones como "construir soberanía alimentaria", "contribuir al cambio al elegir lo que comemos", "generar comunidades" y "apoyar productores locales" (Som Alimentació, 2020). Dada la naturaleza del sistema agroalimentario y su regulación, resulta posible un mayor control de la cadena agroalimentaria mediante prácticas alternativas. Desde este punto de partida, las distintas iniciativas plantean diferentes niveles de autogestión y de participación de las personas consumidoras, lo cual requiere de diversos niveles de compromiso, como se ve en la Tabla 1: por ejemplo, los grupos de consumo buscan una total autogestión, por lo cual parecen atraer a un colectivo más reducido de personas, más 
comprometidas y politizadas. Som Alimentació apunta a un colectivo de personas también movilizado, pero a veces sin la posibilidad o la prioridad para participar más activamente, como también ocurre con la plataforma online. Por otra parte, los mercados de venta directa y los esquemas de cajas parecen referirse a un público que busca más flexibilidad y menos compromiso, aunque también con cierto nivel de conciencia previa. En todos los casos se construyen igualmente relaciones más directas y se da más poder a personas productoras y consumidoras. Se apunta también a la desmercantilización de la alimentación: por ejemplo, en ningún caso se imponen precios a los productores. Además, en todos los casos se trata también de esquemas sin ánimo de lucro.

En segundo lugar, sobre la construcción de redes, observamos también similitudes y diferencias entre los dos nichos. En ambos resulta fundamental, pero mientras que en el ámbito energético existe un proceso más incipiente de construcción de redes, en el caso agroalimentario existen redes más articuladas.

En el nicho de la energía, muchas personas e iniciativas, como es el caso de las seńaladas, llevan compartiendo espacios de trabajo hace tiempo, como la mencionada Plataforma por un Nuevo Modelo Energético. Estos espacios de encuentro han sido fundamentales para fortalecer las organizaciones, compartir análisis y realizar acciones de incidencia política ya sea comunes o que pueden realizar en solitario. No obstante, la inexistencia de un espacio de articulación permanente, consolidado y de referencia puede haber sido un problema para la generación de identidad, discurso común y voz única de cara a las administraciones públicas. En cualquier caso, las iniciativas se ven como complementarias y como parte de un único movimiento y lucha por la energía como bien común.

En el caso agroalimentario, miembros de todas las experiencias señaladas tienen relaciones desde hace tiempo. Destaca la existencia de la Plataforma per la Sobirania Alimentària del País Valencià, un espacio informal de encuentro en el que se comparen análisis y estrategias. Se dan, además, otros muchos espacios puntuales de interacción formal e informal. Estas relaciones han sido clave para el fortalecimiento de las propias iniciativas (como veremos más adelante), para generar un discurso común y para disponer de una cierta interlocución compartida frente a la administración pública. Algunos factores para consolidar el trabajo en red mencionados en las entrevistas serían la existencia de un discurso común aglutinante en torno a las ideas de "soberanía alimentaria" y "agroecología", y el trabajo de personas clave que han facilitado el intercambio entre experiencias y la mediación con las administraciones públicas. Como en el caso de la energía, las distintas iniciativas se consideran en general complementarias y parte de un único movimiento.

En tercer lugar, en relación con los procesos de aprendizaje, en ambos casos y dependiendo de la estrategia y tipo de prácticas de las iniciativas, los aprendizajes han estado orientados especialmente a cuestiones técnicas, legales y normativas o a cuestiones políticas y de organización democrática. Sean más instrumentales o más políticos, se trata en todo caso de aprendizajes relevantes para los comunes urbanos, para la desmercantilización y la autogestión.

En el ámbito energético, vemos que las iniciativas de una mayor escala, como Som Energia, han generado aprendizajes de gran importancia en el primer sentido, 
por ejemplo, para manejarse en un mercado enormemente complejo y permeado por grandes desigualdades de poder. En las otras iniciativas, e igualmente en los grupos locales de Som Energia, también se ha generado otro tipo de aprendizajes, como los de organización y participación democrática.

En el ámbito agroalimentario ocurre algo similar. Por ejemplo, en un sentido más técnico, en Som Alimentació se han generado importantes aprendizajes acerca de las formas legales y organizativas más adecuadas para mantener una cooperativa de gran tamańo con un funcionamiento democrático y asambleario con garantías legales. Los mercados de venta directa han ido buscando (y exigiendo) la forma legal y organizativa apropiada para estabilizar su actividad. En un sentido más político, los grupos de consumo han profundizado mucho en esquemas organizativos y en instrumentos de gestión que les permitan la autoorganización y la práctica de una democracia radical, y ello sin perder eficiencia.

\section{Las formas de crecimiento}

En consonancia con sus estrategias y con las limitaciones de los sistemas en que operan, las distintas iniciativas de los nichos desarrollan diferentes formas de crecimiento.

El ámbito energético, como decíamos, está fuertemente limitado por cuestiones regulatorias y tecnológicas que impiden el desarrollo de iniciativas desde una lógica muy distinta a la dominante. Por ello, el crecimiento de escala lo vemos solo en el caso de Som Energia, que ha apostado por la dinámica de aumento de socios y ha llegado a más de 60 mil en ocho años de vida. Implica la renuncia a ciertos principios (como la plena descentralización en la producción), pero le permite incrementar su visibilidad, importancia y capacidad de incidencia, y apoyar multitud de iniciativas más pequeñas de producción. Para no perder el funcionamiento democrático, la cooperativa ha venido reforzando el rol de los grupos locales para la acción política y movilización en cada territorio. Buena parte de las experiencias crecen por replicación. Es el caso de nuevas iniciativas ciudadanas y también de comercialización, asesoramiento, formación o consultoría sobre energía. Por ejemplo, Aeioluz ha inspirado y apoyado la creación de nuevas cooperativas similares. Aunque escapa al alcance del trabajo, el mecanismo de traslación también se habría dado: el nicho ha generado un debate que parece haber producido cambios en las prácticas energéticas de los actores dominantes, que han incorporado en sus discursos cuestiones como la eficiencia energética y el autoconsumo.

En el nicho de canales alimentarios alternativos también se presentan distintas formas de crecimiento. Algunas iniciativas buscan crecer por replicación. Estas consideran que deben limitar su crecimiento, ya sea por los límites naturales de un espacio (mercados) o por mantener la dimensión fuertemente comunitaria y participativa (los grupos de consumo renuncian a crecer para mantener su funcionamiento radicalmente democrático), pero desean que su experiencia genere iniciativas similares en otros barrios (por ejemplo, todos los grupos de consumo han nacido con el apoyo directo de otros). Som Alimentació opta por el crecimiento de escala. Considera que puede hacerlo incrementando el número de socios o clientes, de tiendas o de canales (por ejemplo, está planteando abrir una tienda online). 
Finalmente, se puede pensar que se han dado importantes procesos de traslación, y que el nicho está influenciando el régimen, si no en los valores o formas, sí en el tipo de productos que se ofrecen (por ejemplo, en supermercados o tiendas que operan en el modelo agroalimentario convencional).

\section{Políticas públicas municipales}

Se han dado varias respuestas a estos procesos desde las políticas locales en los últimos años, aunque las iniciativas demandan una mayor profundización. En todo caso, el despliegue de estas políticas es desigual entre los dos nichos estudiados.

En el ámbito de la energía se han producido, al parecer, numerosas acciones municipales orientadas al apoyo de las iniciativas. Las personas entrevistadas mencionan varias: acciones de visibilización de alternativas; la introducción de la cuestión de la transición energética y de la pobreza energética en el ámbito municipal; o el desarrollo de proyectos de innovación para experimentar sobre autoproducción. Sobre todo, se destaca el esfuerzo público destinado a generar redes y espacios de encuentro de distintos actores. En términos de acciones que permitan el escalado de iniciativas existentes, no se han señalado acciones realizadas, aunque la administración local ha estado valorando iniciativas como la compra de energía a cooperativas, el avance en la autoproducción de energía en edificios públicos, o la creación de una comercializadora de energía local con control social. No se han detectado acciones que puedan identificarse como de protección de iniciativas del nicho.

En el marco de la alimentación, sí se han dado algunas políticas orientadas a la protección de los nichos, tales como los cambios en la regulación municipal para facilitar la creación de mercados de venta directa. En términos de apoyo al desarrollo de las iniciativas, algunas de las personas entrevistadas destacan las acciones destinadas a visibilizar y prestigiar la producción y consumo agroecológico y los canales alimentarios alternativos (campańas orientadas a valorizar el trabajo de agricultores locales, ferias en lugares representativos, promoción de sellos participativos y de producción local, etc.). Además, se destaca el esfuerzo por reducir los recelos del personal funcionario municipal ante la interpretación de normativas que permitan la venta de productos agroecológicos. Sobre políticas orientadas a facilitar el escalado y expansión de iniciativas del nicho, el ayuntamiento ha tomado medidas, por ejemplo, en relación con la gobernanza (se ha generado un Consejo Alimentario Municipal) y la planificación (se ha elaborado una Estrategia Alimentaria Municipal, que hace énfasis en canales alimentarios cortos y alternativos).

Análisis integrado: tendencias y complejidad en la innovación social desde la base Identificamos ahora cinco tendencias e ideas clave a partir del análisis integrado de los aspectos explorados.

En primer lugar, los resultados muestran cómo las iniciativas en ambos sectores plantean visiones y prácticas orientadas a la construcción de comunes urbanos para el derecho a la ciudad en una doble dimensión: político-organizativa y espacial. En el aspecto político-organizativo, los casos generan dinámicas como la implementación de modelos de organización y propiedad que anteponen el bien común al lucro, lo cual profundiza en la desmercantilización; canales cortos de relación directa entre 
productores y consumidores, que permiten la descentralización; nuevos espacios democráticos, que facilitan la autoorganización, la movilización y la transformación personal, etcétera. Todos estos factores, por su parte, tienen impacto en la producción del espacio: las iniciativas activan nuevos mecanismos para la construcción del derecho a la ciudad por sus habitantes. Por ejemplo, la compra de productos agroecológicos, al viabilizar económicamente proyectos productivos agroecológicos locales, permite que suelo periurbano agrícola deje de estar degradado o empleado para una agricultura industrial dependiente de precios internacionales. Los proyectos que gestionan este suelo son con frecuencia cooperativos y, en todo caso, anteponen la producción ecológica y el bien común a la maximización del rendimiento. Lo mismo ocurre con ciertos espacios vacantes, como azoteas comunitarias en las que se instalan paneles fotovoltaicos, que quedan activadas desde la lógica de lo común. Las iniciativas de eficiencia energética generan también otro tipo de relación con la ciudad construida: los habitantes transforman o demandan transformaciones en sus edificios, realizan nuevas operaciones cotidianas sobre ellos (por ejemplo, mediante técnicas bioclimáticas) o toman decisiones más conscientes, que permiten tener más control sobre el uso y consumo de energía. Además, las iniciativas analizadas en ambos sectores están generando nuevos lugares de gestión cooperativa en la ciudad consolidada (como el supermercado de Som Energia), apoyando espacios autogestionados existentes (por ejemplo, el grupo local de Som Energia o los grupos de consumo se reúnen en centros sociales autogestionados existentes), o reivindicando la apropiación ciudadana del espacio público (por ejemplo, los colectivos que impulsan los mercados, que han promovido cambios en la normativa y en su interpretación). Cabe mencionar también que las personas que participan en las iniciativas señaladas transforman sus miradas sobre el espacio que habitan; por ejemplo, sobre el entorno agrícola periurbano o sobre los espacios vacantes.

En segundo lugar, los principios señalados no se presentan con la misma radicalidad en todas las iniciativas. Encontramos dos tendencias en ambos sectores: en un extremo, iniciativas (como los grupos de consumo o algunos de compra colectiva de energía) que generan prácticas más claramente cercanas a la lógica de lo común, pero que tienen menos impacto cuantitativo directo en términos político-organizativos y espaciales: movilizarían un menor número de personas y tendrían un efecto menor en cuanto a apropiación del territorio. Se orientarían a crecer de manera más lenta, sobre todo por replicación. Sin embargo, podrían también estar inspirando otras prácticas menos radicales pero más escalables (por ejemplo, Som Alimentació y uno de los mercados surgen de la experiencia de los grupos de consumo), por lo que también tendrían un importante impacto cuantitativo indirecto. En el otro extremo, tendríamos prácticas que operan de manera más pragmática, como Som Alimentació o Som Energia. Estos casos generan prácticas menos cercanas a la idea de comunes urbanos expuesta, pero tienen más posibilidad de escalado, un mayor impacto territorial (por ejemplo, por la mayor demanda de producción agroecológica local) y crean igualmente procesos de interés para la construcción de comunes, ya que generan espacios de participación y exploran posibilidades de operar con una lógica no mercantilista dentro de la regulación y el mercado. 
En tercer lugar, resulta relevante la complementariedad de prácticas. Las iniciativas proponen esquemas en buena parte complementarios, que llegan a distintos públicos (con distintos niveles de conciencia y compromiso) y se apoyan entre sí. Se puede decir que las prácticas más radicales experimentan permanentemente e inspiran otras más pragmáticas y orientadas a escalar. Las más pragmáticas y no tan estrictamente coherentes con los principios del derecho a la ciudad apoyan a las pequeñas; otorgan mayor visibilidad, legitimidad y capacidad al nicho; y dan la sensación de que un sistema alternativo a mayor escala es posible.

En cuarto lugar, las características del régimen dominante parecen tener una importancia fundamental. El ámbito energético tiene más problemas para la creación de comunes en la ciudad, dada la naturaleza del bien y los límites de la regulación, que obligan a acudir a procesos y tecnologías centralizadas. Con todo, en Valencia estarían operando numerosas iniciativas que aprovechan los márgenes y oportunidades. El sistema alimentario permite mayores niveles de autogestión en la producción, distribución y consumo al margen de las prácticas dominantes, presentes con diferente intensidad en las iniciativas estudiadas.

En quinto lugar, la administración local estaría generando políticas relevantes en la medida en que se adaptan a tipos de iniciativas, estrategias y procesos diferentes. La existencia de redes y de discursos bien articulados por parte de los nichos parece fundamental para avanzar en este sentido. Con todo, se advierten importantes vacíos, especialmente en el ámbito de la energía, con menos recorrido. Parece, en general, haber más dificultad para incentivar el nacimiento de nuevas iniciativas o facilitar su escalado que para apoyar las existentes.

\section{Reflexiones finales}

El marco propuesto ha permitido abordar distintos aspectos de los procesos de innovación social para la construcción de comunes urbanos. Por ejemplo: las limitaciones y ventanas de oportunidad del contexto (cuestiones tecnológicas y regulatorias, entre otras); las visiones comunes existentes y la diversidad de estrategias entre iniciativas de base (estrategias más "intensivas" que generan procesos colectivos más profundos pero minoritarios, o más "extensivas”, más superficiales pero escalables); las relaciones y complementariedad entre iniciativas; o la articulación de distintas políticas ante esta diversidad de estrategias y realidades de las iniciativas.

Sin embargo, los resultados sugieren tensiones y contradicciones en relación con el potencial de los comunes urbanos para movilizar el derecho a la ciudad, que pueden llevar a la remercantilización, la pérdida de autonomía o la renuncia a valores fundamentales. Por ejemplo, pueden darse procesos de cooptación de iniciativas por parte del mercado o de la administración. Por un lado, aspectos superficiales de iniciativas como las señaladas pueden fácilmente ser incorporados por el mercado, limitando su potencial y confundiendo a la ciudadanía. Este es un riesgo observado en múltiples contextos y para sectores como el alimentario y el energético (basta ver los alimentos ecológicos en los supermercados y los sellos de producción verde de las grandes energéticas), de difícil abordaje (Haigh \& Hoffman, 2012). Por otro lado, el apoyo a estas iniciativas y la adopción de su discurso por parte de la 
administración puede ser motivo de desmovilización, como muestra la experiencia de gobiernos progresistas en América Latina que adoptaron el discurso del derecho a la ciudad (Belda-Miquel et al., 2016) o, más recientemente, la experiencia de los nuevos gobiernos municipalistas en distintas ciudades españolas (Fernández, 2018; Gelderloos, 2019). Otro riesgo que sugieren los resultados, observado en el ámbito internacional para casos como los estudiados, es la dificultad por parte de las iniciativas de escalar e incrementar su impacto sin perder la lógica y capacidad transformadoras (Smith et al., 2016).

Los resultados sugieren otras tensiones y contradicciones que no parecen plenamente capturadas por el marco propuesto. Por ejemplo, las relaciones de poder que se dan en el seno de estas iniciativas, o el hecho de que sus miembros son con frecuencia individuos con un nivel socioeducativo medio o alto y experiencia previa en el activismo. Futuras investigaciones podrían refinar el marco para abordar con más detalle estas cuestiones.

En todo caso, el trabajo revela que es prometedor el diálogo entre la literatura sobre derecho a la ciudad y comunes urbanos y la literatura sobre innovación, para explorar los procesos de construcción de nuevos sistemas "desde abajo" en las ciudades, a partir de la lógica de lo común.

\section{Referencias bibliográficas}

Agyeman, J., Bauwens, M., Bernardi, M., Bevolo, M., Bloom, G., Botta, M. \& Ede, S. (2016). The city as commons: A policy reader. Commons Transition Coalition. http://vuir. vu.edu.au/38801/1/city-as-commons.pdf

Andretta, M., Piazza, G. \& Subirats, A. (2015). Urban dynamics and social movements. En D. Della Porta \& M. Diani (Eds.), The Oxford handbook of social movements (pp. 200-218). Oxford University Press. https://doi.org/10.1093/oxfordhb/9780199678402.013.62

APPA [Asociación de Empresas de Energías Renovables]. (2013). Estudio del impacto macroeconómico de las energías renovables en España 2013. https://appa.es/wp-content/ uploads/descargas/Informe_2013_Web.pdf

Belda-Miquel, S., Peris Blanes, J. \& Frediani, A. (2016). Institutionalization and depoliticization of the right to the city: Changing scenarios for radical social movements. International Journal of Urban and Regional Research, 40(2), 321-339. https://doi.org/10.1111/14682427.12382

Blanco, I. \& Gomà, R. (2016). El municipalisme del bé comú. Icaria.

Blanco, I., Gomà R. \& Subirats, L. (2018). El nuevo municipalismo: derecho a la ciudad y comunes urbanos. Gestión y Análisis de Políticas Públicas, 10(20), 14-28. https://doi. org/10.24965/gapp.v0i20.10491

Bollier, D. (2014). Think like a commonier: A short introduction to the life of the commons. New Society Publishers.

Brown, A. (2013). The right to the city: Road to Rio 2010. International Journal of Urban and Regional Research, 37(3), 957-971. https://doi.org/10.1111/1468-2427.12051 
Capellán-Pérez, I., Campos-Celador, Á. \& Terés-Zubiaga, J. (2018). Renewable Energy Cooperatives as an instrument towards the energy transition in Spain. Energy Policy, 123, 215-229. https://doi.org/10.1016/j.enpol.2018.08.064

Castells, M. \& Hlebik, S. (2016). Prácticas económicas alternativas en Barcelona: sobrevivir a la crisis, reinventar la vida. En M. Castells (Coord.), Otra economía es posible: cultura y economía en tiempos de crisis (pp. 226-233). Alianza Editorial.

Castro-Coma, M. \& Martí-Costa, M. (2016). Comunes urbanos: de la gestión colectiva al derecho a la ciudad. EURE, 42(125), 131-153. http://dx.doi.org/10.4067/S025071612016000100006

Corbetta, P. (2003). Metodología y técnicas de investigación social. McGraw-Hill.

Díaz Escobar, C. (2014). El enfoque del decrecimiento como estrategia de empoderamiento y transformación social. Estudio de caso de los grupos de consumo agroecológico de Granada (España). Trabajo Social Global (TSG) - Global Social Work: Revista de investigaciones en intervención social, 4(6), 49-71. https://dialnet.unirioja.es/servlet/ articulo? codigo $=5304713$

Fernández, V. P. (2018). The institutionalization of the right to the city: the Spanish case. En K. Bezdecny \& K. Archer, Handbook of Emerging 21st-Century Cities (pp. 270-290). Edward Elgar Publishing.

Feola, G. \& Nunes, R. (2014). Success and failure of grassroots innovations for addressing climate change: the case of the Transition Movement. Global Environmental Change, 24, 232-250. https://doi.org/10.1016/j.gloenvcha.2013.11.011

Gago, V. \& Pérez, E. G. (2014). Ciudad próspera, ciudad monstruosa: nuevas racionalidades urbanas a partir del caso Indoamericano. Quid 16: Revista del Área de Estudios Urbanos, (4), 66-83. https://publicaciones.sociales.uba.ar/index.php/quid16/article/view/1152

Geels, F. W. (2002). Technological transitions as evolutionary reconfiguration processes: a multi-level perspective and a case-study. Research Policy, 31(8-9), 1257-1274. https:// doi.org/10.1016/S0048-7333(02)00062-8

Geels, F. W. (2010). Ontologies, socio-technical transitions (to sustainability), and the multi-level perspective. Research Policy, 39(4), 495-510. https://doi.org/10.1016/j. respol.2010.01.022

Gelderloos, P. (2019). ¿En qué han fallado los partidos municipalistas en España? Trad. Á. Carvajal. Libre pensamiento, (100), 53-61. https://dialnet.unirioja.es/servlet/ejemplar? codigo $=539070 \&$ info=open_link_ejemplar

Goodman, D., DuPuis, E. M. \& Goodman, M. K. (2012). Alternative food networks: knowledge, practice, and politics. Routledge.

Haigh, N. \& Hoffman, A. (2012). Hybrid organizations: the next chapter in sustainable business. Organizational Dynamics, 41(2), 126-134. https://doi.org/10.1016/j. orgdyn.2012.01.006

Hargreaves, T., Hielscher, S., Seyfang, G. \& Smith, A. (2013). Grassroots innovations in community energy: The role of intermediaries in niche development. Global Environmental Change, 23(5), 868-880. https://doi.org/10.1016/j. gloenvcha.2013.02.008

Harvey, D. (2004). El nuevo imperialismo. Akal. 
Hernández, J. L. S., Penela, A. N., Santos, J. L. A. \& Gutiérrez, L. M. (2017). Regeneración urbana, innovación social y prácticas económicas alternativas en ciudades medias: el barrio del Oeste (Salamanca). Ería: Revista cuatrimestral de geografia, 37(1), 67-82. https://doi.org/10.17811/er.1.2017

Hoppe, T., Graf, A., Warbroek, B., Lammers, I. \& Lepping, I. (2015). Local governments supporting local energy initiatives: Lessons from the best practices of Saerbeck (Germany) and Lochem (The Netherlands). Sustainability, 7(2), 1900-1931. https:// doi.org/10.3390/su7021900

Hossain, M. (2018). Grassroots innovation: The state of the art and future perspectives. Technology in Society, 55, 63-69. https://doi.org/10.1016/j.techsoc.2018.06.008

Kivimaa, P. \& Kern, F. (2016). Creative destruction or mere niche support? Innovation policy mixes for sustainability transitions. Research Policy, 45(1), 205-217. https://doi. org/10.1016/j.respol.2015.09.008

Lefebvre, H. (2003 [1970]). The urban revolution. University of Minnesota Press.

Lincoln, Y. S., Lynham, S. A. \& Guba, E. G. (2011). Paradigmatic controversies, contradictions, and emerging confluences, revisited. En N. Denzin \& Y. Lincon (Eds.), The Sage Handbook of Qualitative Research (pp. 97-128). SAGE Publications.

Marques, P., Morgan, K. \& Richardson, R. (2018). Social innovation in question: The theoretical and practical implications of a contested concept. Environment and Planning C: Politics and Space, 36(3), 496-512. https://doi.org/10.1177/2399654417717986

Mayer, M. (2009). The 'right to the city' in the context of shifting mottos of urban social movements. City, 13(2-3), 362-374. https://doi.org/10.1080/13604810902982755

Miles, M. B. \& Huberman, A. M. (1984). Qualitative Data Analysis: A sourcebook of new methods. SAGE Publications.

Miró, I. (2015). ¿¿Por qué le llamamos innovación a lo que vivimos cómo autogestión? En J. Subirats \& A. García, Innovación social y politicas públicas en España. Análisis de las grandes ciudades (pp. 205-223). Icaria.

Moragues-Faus, A. (2017). Problematising justice definitions in public food security debates: Towards global and participative food justices. Geoforum, 84, 95-106. https://doi. org/10.1016/j.geoforum.2017.06.007

Moulaert, F. (2010). Social innovation and community development. Concepts, theories and challenges. En F. Moulaert, F. Martinelli, E. Swyngedouw \& S. Gonzalez (Eds.), Can neighbourhoods save the city? Community development and social innovation (pp. 4-16). Routledge.

Ornetzeder, M. \& Rohracher, H. (2013). Of solar collectors, wind power, and car sharing: Comparing and understanding successful cases of grassroots innovations. Global Environmental Change, 23(5), 856-867. https://doi.org/10.1016/j. gloenvcha.2012.12.007

Pellicer-Sifres, V., Belda-Miquel, S., López-Fogués, A. \& Boni Aristizábal, A. (2017). Grassroots social innovation for human development: An analysis of alternative food networks in the city of Valencia (Spain). Journal of Human Development and Capabilities, 18(2), 258-274. https://doi.org/10.1080/19452829.2016.1270916

Purcell, M. (2013). The right to the city: the struggle for democracy in the urban public realm. Policy \& Politics, 42(3), 311-327. https://doi.org/10.1332/030557312X655639 
Purcell, M. (2014). Possible worlds: Henri Lefebvre and the right to the city. Journal of Urban Affairs, 36(1), 141-154. https://doi.org/10.1111/juaf.12034

Riutort Isern, S. (2015). Reapropiación popular de la energía en los albores de una transición incierta Una contribución a partir del análisis de caso de Som Energia. (Tesis doctoral inédita). Universitat de Barcelona. http://hdl.handle.net/2445/98395

Sánchez-Herrero, M. (2014). Consecuencias económicas del modelo energético. En C. Romero \& J. V. Barcia, Alta Tensión: Por Un Nuevo Modelo Energético Sostenible, Democrático y Ciudadano (pp. 189-194). Icaria.

Schot, J. \& Geels, F. W. (2008). Strategic niche management and sustainable innovation journeys: theory, findings, research agenda, and policy. Technology analysis \& strategic management, 20(5), 537-554. https://doi.org/10.1080/09537320802292651

Seyfang, G. \& Haxeltine, A. (2012). Growing grassroots innovations: exploring the role of community-based initiatives in governing sustainable energy transitions. Environment and Planning C: Government and Policy, 30(3), 381-400. https://doi.org/10.1068/ c10222

Seyfang, G. \& Longhurst, N. (2013). Desperately seeking niches: Grassroots innovations and niche development in the community currency field. Global Environmental Change, 23(5), 881-891. https://doi.org/10.1016/j.gloenvcha.2013.02.007

Seyfang, G. \& Smith, A. (2007). Grassroots innovations for sustainable development: Towards a new research and policy agenda. Environmental Politics, 16(4), 584-603. https://doi. org/10.1080/09644010701419121

Smith, A., Fressoli, M., Abrol, D., Arond, E. \& Ely, A. (2016). Grassroots innovation movements. Taylor \& Francis.

Som Alimentació. (2020). Filosofia. https://somalimentacio.com/filosofia/

Som Energía. (2020). Quiénes somos. https://www.somenergia.coop/es/quienes-somos/

Subirats, J. \& García, A. (2015). Innovación social y politicas públicas en España. Análisis de las grandes ciudades. Icaria.

Suriñach, R. (2017). Las economias transformadoras en Barcelona. Ayuntamiento de Barcelona.

Xarxa per la Sobirania Energètica. (2018). jTenim energia! Reptes de la transició cap a la sobirania energètica. Icaria. 